\title{
Developing Learning Together - Learning Development Networks and LearnHigher
}

\section{Christine Keenan}

Bournemouth University, UK

This article reflects on the potential of communities and networks, and on the relationship between the Learning Development in Higher Education Network (LDHEN), the Association for Learning Development in Higher Education (ALDinHE) and the LearnHigher CETL.

The LDHEN email discussion group was first set up by John Hilsdon in 2002. The following year, he and Sandra Sinfield established it as a JISCmail list which brought together people from Higher Education institutions across the UK who shared an interest in student learning (Hilsdon, 2004). The development of the LDHEN community provided a place for participants to engage in joint activities and discussions, help each other, and share information - a real 'community of practice' (Wenger, 1998).

The first LDHEN symposium was held at London Metropolitan University in 2003. The title of the conference "Learning Development - What might it be?" provided plenty of food for thought. It also set off discussion and debate about the nature of learning development. There was another very happy outcome of the first symposium. Bill Miller, (then of Liverpool University), came along with Jill Armstrong, (then of Liverpool Hope University and the LTSN Generic Centre, forerunner to the HEA). They were concerned that there seemed to be a great number of practitioners often working on their own, developing ideas and resources to benefit their own students learning. They suggested setting up a "One Stop Swap Shop" for the exchange of resources and shared practice. A number of people at the Symposium agreed with this idea, and joined forces to find a way to bring the idea to reality.

This new partnership of individuals from within the LDHEN network, representing sixteen institutions, began exploring ideas for a collaborative venture to facilitate the 
sharing of learning materials and exchange of ideas. Finding external funding was crucial to getting the idea off the ground.

A great opportunity presented itself in 2004 with the announcement by the Higher Education Funding Council for England of the setting up of Centres for Excellence in Teaching and Learning. The funding available was significant and the proposed five year time scale offered an opportunity for the partnership to set about writing a bid which took the idea of a swap shop of learning resources and added the dimensions of peer-review, evaluation and underpinning research.

A strength of the bid submitted to HEFCE was that it developed from an existing network of people within the UK Learning Development community. Responsibility for writing and proof-reading the four sections of the Phase One bid was shared between members of the group and the bid was submitted.

A particular strength identified by the phase one reviewers was that the overall theme and development of learning resources would be underpinned by a research and evaluation base well-matched to the collaborative strengths of the partnership. Reviewers recognised the considerable scope for further growth and impact and saw the potential for impact across the whole sector. Importantly, the reviewers commented positively on the existing links with the growing national network of learning developers.

In 2005 the LearnHigher CETL was launched with Liverpool Hope as the lead institution. Each of the 16 partners received an amount of capital money which has been spent on learning spaces in their institutions - examples of which are described on the website http://www.learnhigher.ac.uk under LearnHigher spaces. Annual recurrent funding allows each partner some flexibility in how their commitment to the development of LearnHigher is managed, and provides for the development of resources in their learning area. A pot of money has also been made available to fund collaborative development projects which are also described in reports available from the website. 
LearnHigher now offers a range of reviewed, excellent resources online to the learning development community and the sector as a whole. It is also working to raise the profile of learning development across the sector. LearnHigher is developing practitioner-led inquiry into the key questions about how, when and why students use learning resources, and supports more specialised research, development and evaluation activities within the learning topic areas.

Meanwhile, the LDHEN network has continued to grow and is one of the liveliest JISCmail discussion groups. With more than 360 subscribers currently and some 200 HE institutions represented, the network continues to support a highly active community of practice for learning development.

The strength of the community and the powerful support mechanisms that the list has provided since 2002 has enriched the practice and experience of many members who undoubtedly feel less as a result. In 2008 Cash \& Hilsdon presented a paper to the SRHE conference in Liverpool, arguing that: "The network provides a medium through which the discourses and practices of 'learning development' (LD) are being described, argued, theorised and refined." Their findings were based on the content of 1527 e-mails under 565 subject headings posted on the LDHEN list between January 2003 and December 2007. Three overall themes were identified; mapping the staffing and provision of learning development in institutional contexts, debating issues related to LD principles and practice, and, sharing and commenting on resources related to teaching and student learning. The annual symposia also provide learning developers with the opportunity for scholarly reflection on learning development and practice and the new journal demonstrates growth, confidence and a legitimacy of our field of practice.

The growing confidence of the community is ensuring that the role of learning developer has a higher profile within institutions and the community is increasingly influencing institutional policies. On this basis, at the $4^{\text {th }}$ LDHEN Symposium in 2007, it was decided to establish the Association for Learning Development in Higher Education. As stated on the association's website, a key theme for members is "commitment to the development of student learning and the provision of opportunities for students to develop their skills for study and their awareness of 
academic practices" (ALDinHE, 2007). In an article announcing the launch in the Times Higher Education Supplement, it was proposed that a key aim would be to "... contribute to a renewal of interest in promoting learning at university in its contemporary, democratic contexts, and to aspirations for increasingly accessible higher education" (Hilsdon 2007). Membership of ALDinHE offers the opportunity to strengthen our voice and contribute to the development of policies promoting learning development, and CPD opportunities for staff.

LearnHigher is now in the penultimate year of funding and thoughts are turning to continuation once the funding ceases. A working party drawn from the LearnHigher and ALDinHE Steering Groups has been tasked with looking into the feasibility of continuing the work of the CETL under the auspices of the Association. Proposals to achieve this will be presented to the membership of ALDinHE at the next AGM in April 2009.

The idea that the uniquely collaborative concept of LearnHigher should continue is, of course, appealing to those of us who have already been involved in it. Furthermore, to re-launch LearnHigher in future as part of the Association, and to offer the possibility that new partnerships can develop within it, would be especially welcome. The continuing discussions supported by the LDHEN network; the scholarly contributions developed through our symposia - and now here, via the Journal of Learning Development in Higher Education - combined with the resource development activities of LearnHigher; all these related initiatives represent the flowering of a community who just want to continue developing and learning together.

\section{References}

Cash, C. and Hilsdon, J. (2008) Buried Treasures in a Virtual Community Chest. Paper presented to the SRHE conference, Liverpool 2008. ONLINE http://www.srhe.ac.uk/conference2008/papers/0292-John-Hilsdon CarolineCash.doc 
Hilsdon, J. (2004) Learning development in higher education network: an emerging community of practice? Educational Developments 5.3, Birmingham: SEDA.

Hilsdon, J. (2007) A Learning Renaissance? Times Higher Education Supplement, 6th July 2007

Wenger, E. (1998) Communities of practice: learning, meaning, and identity. Cambridge: Cambridge University Press.

Christine Keenan is a Learning and Teaching Fellow at Bournemouth University 